\title{
The Genetic Relevance of Human Induced Pluripotent Stem Cell-Derived Microglia to Alzheimer's Disease and Major Neuropsychiatric Disorders
}

\author{
Robert R. Butler IIII, $\quad$ Alena Kozlova ${ }^{a, b} \quad$ Hanwen Zhang $^{a} \quad$ Siwei Zhang ${ }^{a, b} \quad$ Michael Streit $^{a}$

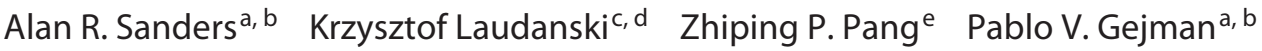 \\ Jubao Duan ${ }^{\mathrm{a}, \mathrm{b}}$ \\ ${ }^{a}$ Center for Psychiatric Genetics, NorthShore University HealthSystem, Evanston, IL, USA; ${ }^{b}$ Department of Psychiatry \\ of Behavioral Neuroscience, The University of Chicago, Chicago, IL, USA; ' Department of Anesthesiology and Critical \\ Care, The University of Pennsylvania, Philadelphia, PA, USA; ${ }^{d}$ Institute for Translation Medicine and Therapeutics, \\ The University of Pennsylvania, Philadelphia, PA, USA; ${ }^{e}$ Department of Neuroscience and Cell Biology and Child \\ Health Institute of New Jersey, Rutgers University, New Brunswick, NJ, USA
}

\section{Keywords}

Human induced pluripotent stem cells · Microglia •

Alzheimer's disease · Schizophrenia · Psychiatric disorder ·

Genetic relevance $\cdot$ Transcriptomic

\begin{abstract}
Microglia are the primary innate immune cell type in the brain that have been implicated in the pathogenesis of several neurodegenerative and neuropsychiatric disorders, most notably Alzheimer's disease (AD) and schizophrenia. Microglia generated from human induced pluripotent stem cells (hiPSCs) represent a promising in vitro cellular model for studying the neuroimmune interactions involved in these disorders. Among several methods of generating hiPSC-derived microglia (iMG) - varying in duration and resultant purity - a recent protocol by Brownjohn et al. [Stem Cell Reports. 2018 Apr;10(4):1294-307] is particularly simple and efficient. However, the replicability of this method, transcriptomic similarity of these iMG to primary adult microglia, and their genetic relevance to disease (i.e., enrichment of
\end{abstract}

() 2019 S. Karger AG, Basel disease risk loci in genes preferentially expressed in these cells) remains unclear. Using two hiPSC lines, we demonstrated that Brownjohn's protocol can rapidly generate iMG that morphologically and functionally resembled microglia. The iMG cells we generated were found to be transcriptionally similar to previously reported iMG, as well as fetal and adult microglia. Furthermore, by using cell type-specific gene expression to partition disease heritability, we showed that iMG cells are genetically relevant to AD but found no significant enrichments of risk loci of Parkinson's disease, schizophrenia, major depressive disorder, bipolar disorder, autism spectrum disorder, or body mass index. Across a range of neuronal and immune cell types, we found only iMG, primary microglia, and microglia-like cell types exhibited a significant enrichment for AD heritability. Our results thus support the use of iMG as a human cellular model for understanding $A D$ biology and underlying genetic factors, as well as for developing and efficiently screening new therapeutics.

(c) 2019 S. Karger AG, Basel

Robert R. Butler III and Alena Kozlova contributed equally to this work.

Jubao Duan

Center for Psychiatric Genetics, NorthShore University HealthSystem

1001 University Place

Evanston, IL 60201 (USA)

E-Mail jduan@uchicago.edu 


\section{Introduction}

Alzheimer's disease (AD) is an age-related neurodegenerative disease that is characterized by a cognitive decline, with histological hallmarks including the deposition of amyloid-beta $(A \beta)$ plaques, the formation of neurofibrillary tangles, and neuroinflammation [1]. Genome-wide association studies (GWAS) on AD have identified more than 20 risk loci [2-5]. Putative AD-relevant genes near GWAS loci are highly expressed in immune-related tissues and cell types, in particular microglia (MGL) [6, 7]. Furthermore, recent single-cell RNAseq profiling of an AD mouse model defined subtypes of MGL associated with different disease stages (i.e., homeostatic MGL, stage-1 trem2-independent disease associated MGL [DAM], and stage-2 trem2-dependent DAM) [8]. Given the limited overlap of transcriptomic profiles between humans and mice [9], studying AD genetic risk factors in human MGL cell models is important to understanding $\mathrm{AD}$ pathogenesis.

MGL are the primary innate immune cells in the brain. Studying human MGL is understandably challenging due to the scarcity of living human brain tissue for research, or cadaver tissue artifacts. Generating in vitro human MGL from a renewable resource such as human induced pluripotent stem cells (hiPSCs) provides an important alternative approach. MGL originate from yolk sac macrophage progenitors during primitive hematopoiesis [10, 11]. These primitive macrophages then migrate into the developing brain, become microglial progenitors, and finally mature MGL. They then function as immune cells, facilitate brain development, and modulate synaptic activity $[12,13]$. To mimic this unique developmental process, different methods have been recently developed to generate induced MGL (iMG) from hiPSCs [14-19]. These protocols differ in the use of different specific growth factors, co-culturing with other types of neuronal cells, starting from embryoid bodies (EBs) or monolayer cell culture, purity of the derived iMG, and the length of the differentiating time. Most recently, Brownjohn et al. [19] developed a simple method that generates functional iMG in large numbers with near $100 \%$ purity within 20-30 days. It starts from EB-released primitive macrophage precursors (PMPs) [19]. Because the EB-based approach historically lacks reproducibility, the replicability of this protocol remains to be independently tested. Moreover, it has not been demonstrated whether the resultant iMG cells are AD-relevant beyond their molecular and transcriptomic resemblance to primary microglial cells, an essential component for using iMG cells as a val- id in vitro cellular model for understanding AD biology. This is a valid point since some MGL emerge from primitive EB precursors, but that knowledge is still evolving. Finally, although complement C4 (C4A) has been implicated in schizophrenia (SCZ) GWAS as a strong genetic risk factor and mechanistically linked to MGL-mediated abnormal synapse pruning $[20,21]$, it remains unknown whether iMG cells are a genetically relevant cell type for SCZ.

Here, we first replicated the results reported by Brownjohn et al. [19] and found that the transcriptomic profiles of our iMG were similar to that of previously reported iMG $[15,19]$, as well as fetal and adult MGL. By partitioning disease heritability of GWAS risk loci proximal to genes specific to each cell type, we also demonstrated that iMG were a significantly enriched cell type for AD risk as were primary adult and fetal MGL - indicating the relevance of $\mathrm{iMG}$ to $\mathrm{AD}$. We compared this to enrichments for disease risk loci of Parkinson's disease (PD) and 4 other major psychiatric disorders - SCZ, major depressive disorder (MDD), bipolar disorder (BIP), and autism spectrum disorder (ASD). Our results provide key support for using hiPSC-derived iMG as a human cellular model to study the molecular/cellular mechanisms and genetic risk factors of $\mathrm{AD}$.

\section{Results}

\section{Replicability of Brownjohn's Protocol [19] to Generate} iMG

We selected two hiPSC lines (CD11 and CD21) well characterized for pluripotency and absence of cryptic chromosomal abnormalities (Fig. 1a, online suppl. Fig. 1a, b; see www.karger.com/doi/10.1159/000501935 for all online suppl. material). As expected, EBs were formed within 4 days after initiation of hiPSC differentiation, and PMPs were continuously produced in suspension beyond 5 days (Fig. 1b). We found that a sufficient amount of PMPs were released between days 14 and 32, with the greatest numbers of PMPs being produced between days 14 and 19. The CD11 iMG were highly pure, with multiple cultures originating from the different plated fractions of PMPs, extending 30 days from the earliest iMG identification at day 24 (Fig. 1a). However, for the CD21 line, batches beyond the first collection of PMPs gave rise to impure iMG cultures, with non-iMG cells increasing in concentration (Fig. 1b). The CD21_3 heterogeneous culture, generated from PMPs collected at day 23, harbored 50\% non-iMG cells, and CD21_4 collected at day DOI: $10.1159 / 000501935$
Butler III et al. 


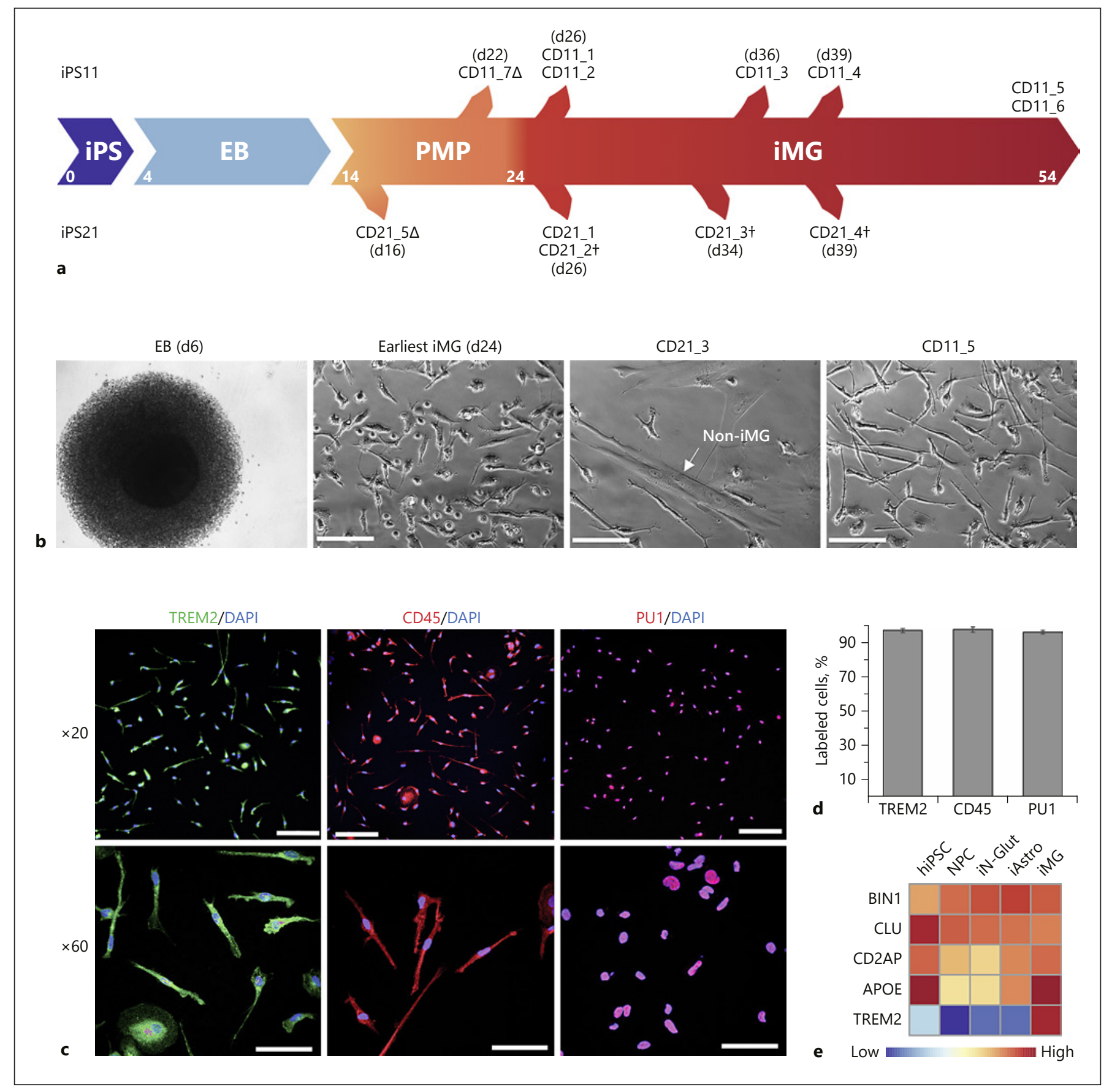

Fig. 1. Generation of hiPSC-derived microglia (iMG). a hiPSC lines iPS11 and iPS21 are differentiated to microglia lines CD11 and CD21, respectively, via embryoid bodies (EB) and primitive macrophage precursors (PMP) over a period of 24 days. PMPs are produced continuously in culture and are terminally differentiated into microglia when required. CD21 and CD11 samples with a delta $(\Delta)$ denote iPMP samples and a dagger $(\dagger)$ denotes mixed samples containing non-iMG cells. b DIC images of EB at day 6 (far left), iMG cells at day 24 (near left, CD11_5), and more mature microglia cells at day 44 (far right, CD11_5). Some of the iMG cultures also had non-iMG cells (near right, CD21_3), which mor- phologically resembled fibroblasts. Scale bars, $500 \mu \mathrm{m}$. c Immunofluorescence staining of iMG from line CD11 at day 24 shows expression of microglial/macrophage markers TREM2, CD45, and PU1. Scale bars in $\times 20$ images, $100 \mu \mathrm{m}$; scale bars in $\times 60$ images, $50 \mu \mathrm{m}$. d The majority of the iMG cells from line CD11 show expression of microglial/macrophage markers TREM2, CD45, and PU1 $(n=4-5)$. e Expression by qPCR of AD risk genes (BIN1, CLU, CD2AP, APOE, TREM2) in different cell types (normalized to GAPDH). iAstro, hiPSC-derived astrocytes; iN-Glut, hiPSCderived glutaminergic neurons; NPC, neural progenitor cells.
Genetic Relevance of iMG to AD and Major Neuropsychiatric Disorders
Mol Neuropsychiatry 2019;5(suppl 1):85-96 DOI: $10.1159 / 000501935$ 
39 were $100 \%$ non-iMG. These non-iMG cells morphologically looked like fibroblasts, which was later confirmed as fibroblast-like by transcriptomic analysis.

Regardless, we were able to successfully produce PMPs and iMG for both lines within the expected time frame, as characterized by immunofluorescence staining of macrophage/MGL-specific markers TREM2, CD45, and PU1 (Fig. 1c, d). Samples with pure iMG by immunofluorescence staining were further tested by qPCR for MGL-specific markers (Fig. 1e, online suppl. Table 1). The iMG showed high-level expression of $\mathrm{AD}$-associated genes, TREM2 and APOE (Fig. 1e). Thus, we were able to confirm the overall transferability of the Brownjohn's iMG protocol [19], producing preliminarily identified iMG. However, careful monitoring for non-iMG cell encroachment is needed, because in some occasions, fibroblastlike cells may be observed in iMG culture (a similar observation has also been observed in by the authors of the original protocol [19]; personal correspondence).

\section{Functional Validation of hiPSC-Derived $I M G$}

We next examined whether the hiPSC-derived iMG were functionally similar to MGL. One of the essential functions of MGL is to phagocytose exogenous pathogens and cellular debris [22]. Using $\mathrm{pH}$-dependent fluorescence imaging, we showed that iMG effectively phagocytosed pHrodo E. coli, and that phagocytosis continued over time. Thirty minutes after adding pHrodo E. coli to iMG, we detected fluorescence signal inside the cells that increased rapidly through $150 \mathrm{~min}$ (Fig. 2a-c). This process is sensitive to cytochalasin $\mathrm{D}$, an inhibitor of actin polymerization, and when added to the culture prior to the pHrodo E. coli, iMG cells lost the ability to phagocytose bioparticles (Fig. 2d). Thus, our iMG cells engage in pathogen-responsive phagocytic behavior that is a hallmark of MGL.

\section{Transcriptomic Similarity of iMG and Fetal/Adult MGL}

To examine how our iMG cells compare to previously reported iMG cells, fetal and adult MGL, we first performed a principal component analysis of transcriptomic profiles from these and other non-microglial cells (Fig. 3a, online suppl. Table 2). The two hiPSC lines used to generate our iMG and neural progenitor cells were clustered together with other hiPSC lines that were previously used [15], suggesting a comparable quality of source hiPSC lines between our current study and previous reports. Our high quality iMG samples (CD11_1-6, CD21_1) were clustered together with previously reported iMG as

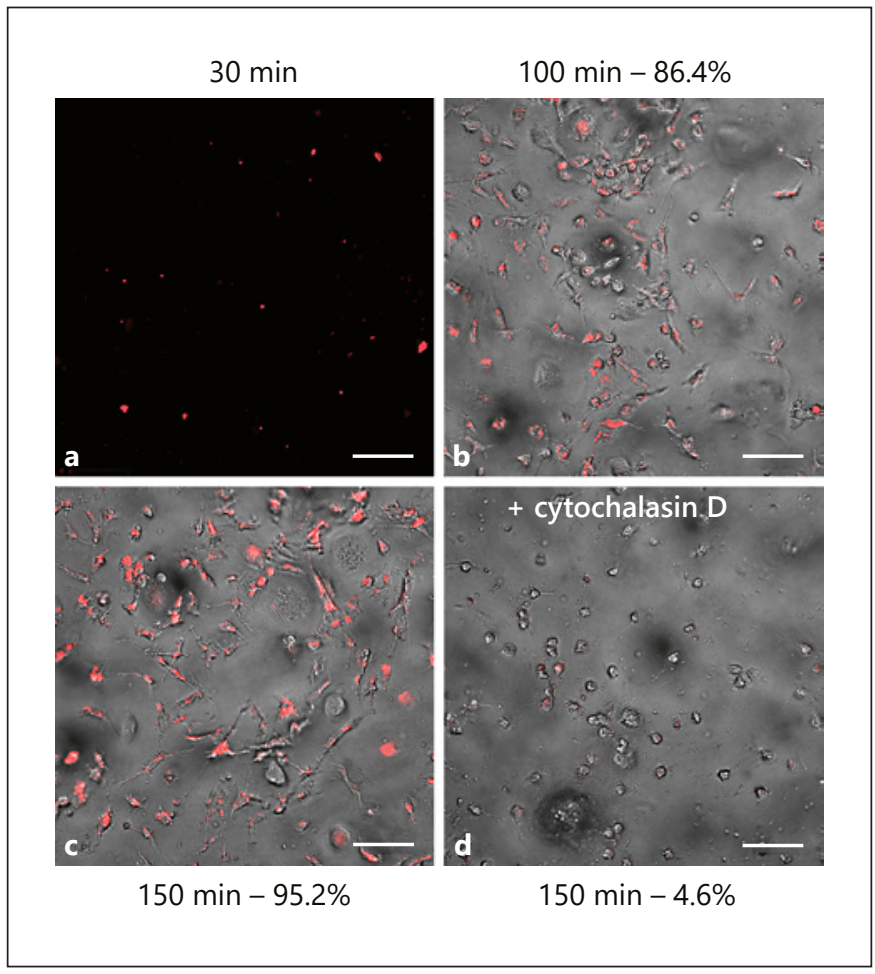

Fig. 2. hiPSC-derived microglia (iMG) phagocytic activity is similar to primary microglia. Fluorescence imaging of day-27 iMG (ac) showing time-dependent phagocytosis of pHrodo labeled $E$. coli, colored red after ingestion. $\mathbf{d}$ This process is inhibited by the addition of cytochalasin D. Percentages are of pHrodo-positive cell counts. All scale bars, $100 \mu \mathrm{m}$. Bright-field unavailable at $30 \mathrm{~min}$.

well as cultured fetal and adult MGL $[15,19]$. PMPs from our current study (CD11_7 and CD21_5) and from previous reports were interspersed with iMG and fetal/adult MGL samples. Overall, PMPs, iMG, and MGL samples were well separated from other cell types; the next closest cell type in the first principal component, monocytes (MC), being effectively separated in the second component (Fig. 3a). These observations were further corroborated by pairwise Poisson distance, with the hierarchical heatmap clustering of gene expression showing a similar grouping of samples (Fig. 3b).

Interestingly, our transcriptomic analyses showed that the observed "mixed" iMG samples, CD21_3 and CD21_4, with heavy and almost total fibroblast-like cell overgrowth, clustered together with fibroblast cells, while the sample with a less concentrated fibroblast-like population (CD21_2) was located in between the fibroblasts and iMG (Fig. 3a). The pairwise clustering heatmap also confirmed the CD21_3 and CD21_4 to be fibroblast-like, with the CD21_2 group showing an intermediate pheno-
Butler III et al. 


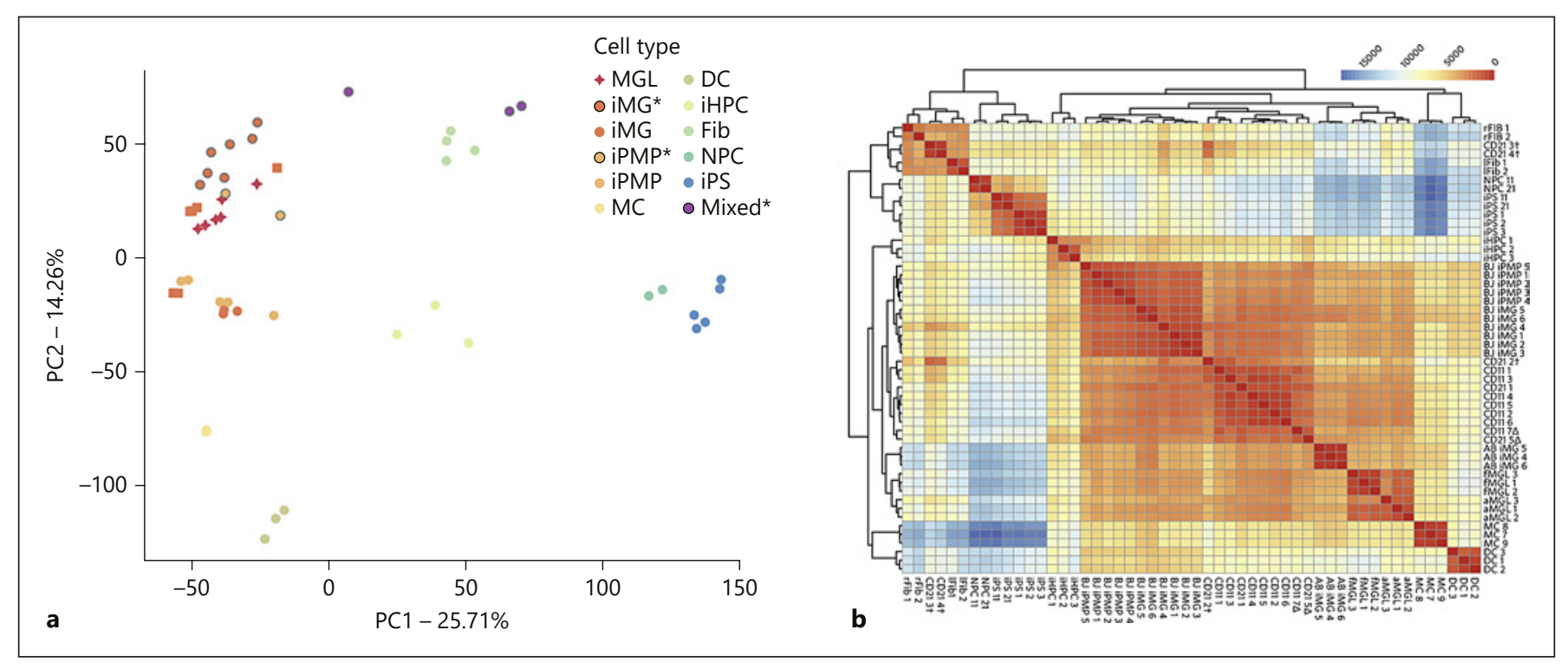

Fig. 3. Transcriptional profiling of hiPSC-derived microglia (iMG). a The first two principal components of various human cellular lineages compared to induced microglia and primitive macrophage precursors. Transcriptomic profiles are assessed on 15,469 quantile-normalized genes, which are differentially expressed across all groups. Samples generated in this study are denoted with an asterisk and grey outlines. DC, dendritic cells; Fib, fibroblasts (lung and retinal tissue derived); iHPC, induced hematopoietic progenitor cells; iMG, induced microglia (squares denote samples from Brownjohn et al. [19], circles denote samples from

type, distinct from the MGL cell types it was clustered with (Fig. 3b). Taken together, the comparative analysis of the transcriptomic profiles of our iMG and other cell types indicate that our iMG cells are indistinguishable from previously reported iMG and adult/fetal MGL at the transcriptomic level.

\section{iMG Are Enriched for AD GWAS Loci, but Lack Enrichment of Major Neuropsychiatric Disease Risk Loci}

MGL have been implicated as a major cell type involved in $\mathrm{AD}$ disease progression [6-8]. However, whether hiPSC-derived iMG cells are also genetically relevant to $\mathrm{AD}$ has not previously been evaluated. We first examined the expression similarity of a set of AD-associated genes [17] between different cell types including our iMG (Fig. 4a). We found that most $A D$ risk genes showed a similarly high level of expression in iMG and fetal/adult MGL, but not in other non-MGL cell types. Hierarchical clustering using expression from these 31 genes also showed that iMG cells were clustered together with fetal/ adult MGL cells. As expected, TREM2 expression is high-
Abud et al. [15]); iPMP, induced primitive macrophage precursors; iPS, induced pluripotent stem cells; MC, monocytes; MGL, primary microglia (adult and fetal); Mixed, contaminated iMG cell lines; NPC, neural progenitor cells. b Pairwise Poisson distance between raw protein-coding gene counts. CD21 and CD11 samples are those generated in this study, with a delta $(\Delta)$ denoting iPMP samples and a dagger $(\dagger)$ denoting mixed samples. $A B$, Abud et al. [15] iMG samples; aMGL, adult microglia; BJ, Brownjohn et al. [19] iMG/iPMP samples; fMGL, fetal microglia; lFib, lung tissue-derived fibroblast; $\mathrm{rFib}$, retinal tissue-derived fibroblast.

ly specific to iMG and fetal/adult MGL. APOE also shows highest expression in iMG and MGL.

To further evaluate whether iMG is a relevant cell type for AD genetic risk, we used LDSC and MAGMA [23-25] to partition heritability of AD GWAS loci near genes specifically expressed in each cell type. This approach has been recently used to estimate disease risk enrichment for each cell type based on genes expressed with high specificity [26], most notably in identifying the SCZ-relevant neuronal cell types [27]. A normalized metric of cell type specificity of gene expression was calculated for each gene using EWCE (Fig. 4b) and subsequently used to rank genes for each cell type. In order to include a broad range of neural and immune cell types that can help define cell type-specific expression, we incorporated additional single-cell RNA-seq data [28] (online suppl. Table 3) to our current dataset and used replicates of bulk RNA-seq for comparison. We found that the distributions of gene specificity values for bulk and single-cell RNA-seq data were comparable, which suggests the normalization and filtering of EWCE produced a cohesive dataset for LDSC and MAGMA analyses. 
LDSC analysis of AD GWAS loci around the top 20\% of genes (by specificity) found enrichment for all MGL, MGL-like cell types, and MCs (Fig. 4c); with single-cell RNA-seq MGL and adult MGL further showing a similar enrichment regardless of sequencing method. Consistent with a recent study showing genome-wide significant $A D$ risk loci plausibly operate in peripheral MC [29], we observed here that $\mathrm{MC}$ showed enrichment for AD risk similar to that of the iMG cells (Fig. 4c, online suppl. Fig. 2). Using narrower gene windows of $20 \mathrm{~kb}$ and $50 \mathrm{~kb}$, we observed even higher enrichment of our iMG Koz cell type than in Figure 4 ( $\alpha<0.004$ in both instances), but chose the default $100 \mathrm{~kb}$ window as it showed best enrichment across all MGL and MGL-like cell types (online suppl. Fig. 2). For other tested GWAS traits (SCZ, PD, MDD, BIP, ASD, body mass index [BMI]) (Fig. 4d), we observed expected significant enrichment of SCZ and BIP GWAS signals in neurons and a lesser enrichment of PD, MDD, and BMI GWAS loci. Oligodendrocytes also showed a mild enrichment for SCZ GWAS loci. Despite the known strong SCZ genetic risk factor complement C4 (C4A) and its associated MGL function in abnormal synapse pruning [20], we did not observe significant enrichment of SCZ GWAS signals in iMG or MGL-like cell types, which is consistent with a previous study [27]. Nor did we observe enrichment of GWAS signals in iMG or MGL-like cell types for BMI and PD GWAS datasets, as expected due to no known involvement of MGL.

We next evaluated the cell type-specific GWAS enrichment with a different tool, MAGMA. The MAGMA cell typing method makes use of linear regression based on the specificity score, allowing the use of all the expressed genes instead of a strict cutoff. Accordingly, we saw an overall higher significance of enrichments in relevant cell types for AD and other GWAS trait sets (Fig. 4d). MAGMA enrichments for AD achieved Bonferroni sig-

Fig. 4. Genetic relevance of hiPSC-derived microglia (iMG) to Alzheimer's disease (AD). a Quantile-normalized RNA expression in 31 genes associated with $\mathrm{AD}$ risk (Haenseler et al. [17]). Columns are individual samples annotated above by cell type. CD21 and CD11 samples are those generated in this study, with a delta $(\Delta)$ denoting induced primitive macrophage precursor (iPMP) samples and a dagger $(\dagger)$ denoting mixed samples (contaminated iMG cell lines). AB, Abud et al. [15] iMG samples; aMGL, adult microglia; BJ, Brownjohn et al. [19] iMG/iPMP samples; DC, dendritic cells; Fib, fibroblasts (lung and retina derived); fMGL, fetal microglia; iHPC, induced hematopoietic progenitor cells; iPS, induced pluripotent stem cells; lFib, lung tissue-derived fibroblast; MC, monocytes; MGL, primary microglia (adult and fetal); NPC, neural progenitor cells; rFib, retinal tissue-derived fibroblast. b Distributions nificance $(\alpha=0.05 / 9$ distinct cell types $\times 7$ GWAS sets $=$ 0.00079) for the MGL, MGL-like, and MC cell types that also showed strongest enrichment of AD loci with LDSC (Fig. 4c). As with LDSC, SCZ GWAS loci were significantly enriched in neurons, but also had significant enrichment in oligodendrocytes, astrocytes, and neural stem cells. MAGMA also gave a non-significant enrichment of AD in astrocytes, which was undetected in LDSC (Fig. 4c). MAGMA showed enrichment of BMI loci in the neuron cell type consistent with previous analysis [26], but also showed significant enrichment in neural stem cells, oligodendrocytes, astrocytes, and endothelial cells (Fig. 4d). PD demonstrated a low level of enrichment across a majority of these cell types without a significant cell type, similar to previous findings [27]. MDD and BIP both showed significant enrichment of disease loci in neurons and oligodendrocytes, and with a lesser significant enrichment in neural stem cells, which is largely consistent with previous observations [26, 27]. It is noteworthy that for ASD that was not previously examined for genetically relevant cell types, we found significant GWAS enrichment in neurons and oligodendrocytes. Taken together, the results from LDSC and MAGMA analyses are overall consistent, showing microglial-specific enrichment of preferentially gene expression of $\mathrm{AD}$ genetic risk loci, but not of the risk loci of SCZ and several other psychiatric disorders.

\section{Discussion}

Multiple attempts with a variety of methods have been made to derive iMG from hiPSCs with mixed results [1419]. We provide evidence that hiPSC-derived iMG share similar transcriptomic profiles with adult MGL and are genetically relevant to AD. Here, using a recently devel-

of gene specificities for each cell type, colored by decile. Single-cell RNA-Seq cell types from Darmanis et al. [28] are highlighted in navy. Ast, astrocytes; Endo, neural endothelial cells; Neu, neurons; Olig, oligodendrocytes; scMGL, single-cell microglia. c AD GWAS loci (Jansen et al. [6]) enrichment for LDSC and MAGMA across cell types, with a Bonferroni threshold at $\alpha=0.05 /(9$ cell types $\times 7$ GWAS traits). d A heatmap of LDSC (left) and MAGMA (right) GWAS enrichment significance for AD, schizophrenia (SCZ; PGC SCZ2 [56]), Parkinson's disease (PD; Nalls et al. [55]), major depressive disorder (MDD; Wray et al. [57]), bipolar disorder (BIP; Stahl et al. [58]), autism spectrum disorder (ASD; Grove et al. [59]), and body mass index (BMI; Yengo et al. [60]) across multiple cell types. Enrichments with Bonferroni-adjusted significance shown with bold borders.
(For figure see next page.)
Butler III et al. 

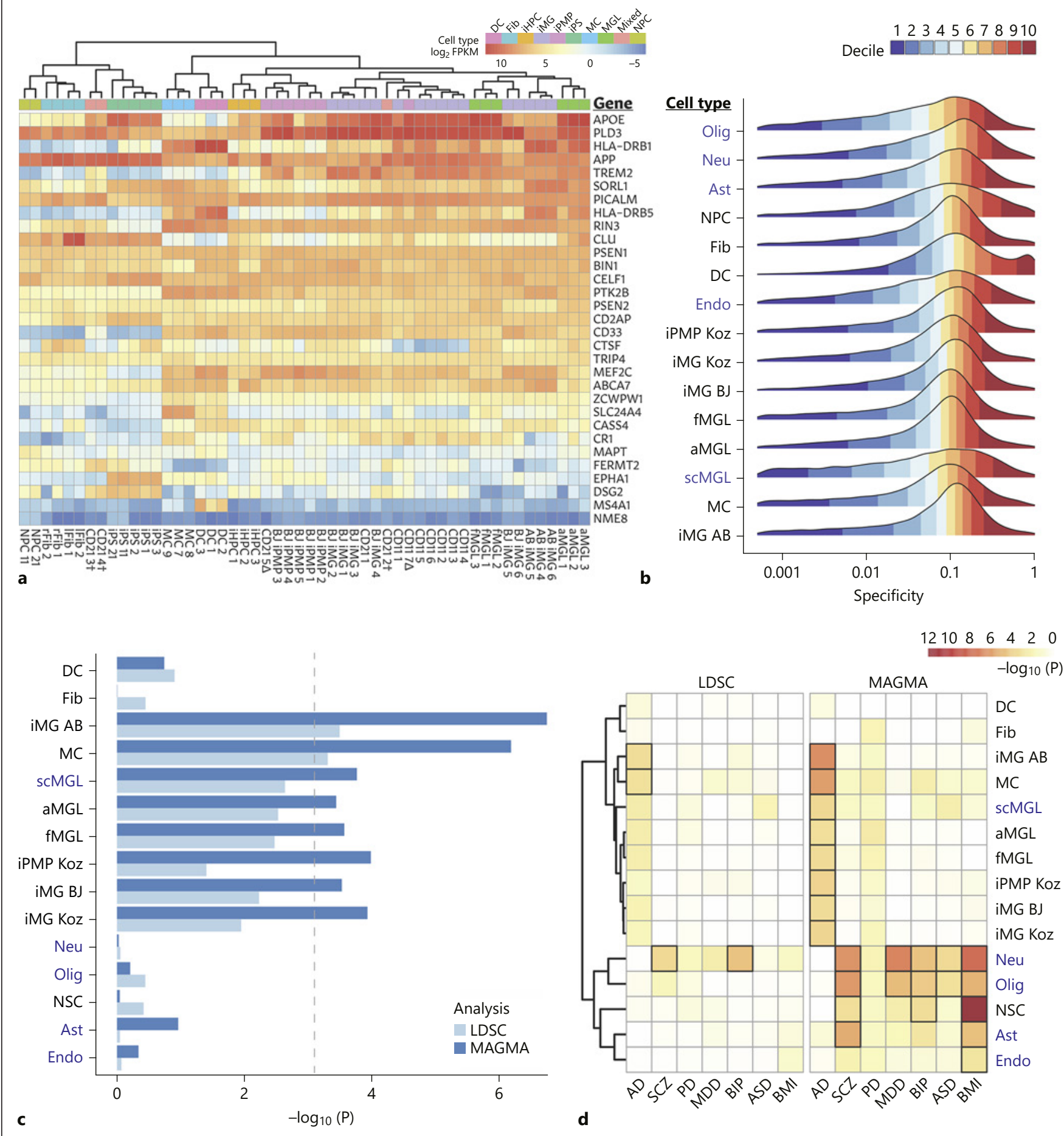
oped and a simple protocol [19], we demonstrated its replicability by generating congruent results. The iMG exhibited morphological and molecular characteristics of MGL and were transcriptionally indistinguishable from adult MGL. We further showed that iMG from hiPSCs and adult MGL are similarly enriched for common AD heritability, but not for SCZ or some other major psychiatric disease risk GWAS loci, which has not been previously demonstrated.

$\mathrm{AD}$ is a polygenic disorder, with possibly hundreds of risk genes each contributing a small heritability. As a conceptual extension of the polygenic model, the omnigenic model for complex disorders recently hypothesized that most genes, if not all, expressed in a disease-relevant cell type may contribute to disease risk [30]. Thus, identifying a disease-relevant cell type will be a key step for dissecting the genetic causality for $\mathrm{AD}$. Using two leading GWAS heritability partitioning toolsets, we have shown that genes expressed in hiPSC-derived iMG were significantly enriched for $\mathrm{AD}$ risk loci (Fig. 4c, d), with a significance level that was similar to that of multiple other MGL sets and MC. Although heritability enrichment analysis is sensitive to some chosen parameters such as the length of flanking genic regions and the metric of gene specificity (online suppl. Fig. 2, 3), the overall cell type-specific enrichments of AD heritability agreed across both methods, pointing to the comparable performance of iMG and MGL. The observed shared enrichment pattern of AD heritability in iMG and MGL cell types was consistent with their expression profiles for previously identified $\mathrm{AD}$-associated genes (Fig. 4a). Taken together with their overall transcriptomic similarity (Fig. 3), our results suggest that hiPSC-derived iMG cells are a genetically relevant cellular model for studying genetic effects of AD risk factors.

Genetically relevant iMG can help to infer the putatively functional causal AD GWAS risk variants and help elucidate their effects in a human cellular model. Because most AD GWAS risk variants are in the noncoding part of the genome, putatively causal variants most likely affect gene expression via altered chromatin accessibility to transcription factors, and modification of regulatory regions. In this regard, open chromatin region profiles of hiPSC-derived iMG cells, like in hiPSC-derived excitatory neurons for SCZ, may help prioritize the putatively functional GWAS risk variants that may alter chromatin accessibility and transcriptional gene networks [31, 32].

We acknowledge the limitations of our results. First, although we were able to replicate Brownjohn's method [19], we also found that some differentiating iMG cul- tures develop persistent fibroblast-like impurities of unclear origin, requiring careful screening of cultures to prevent contamination. Second, given the cell lines used in this study were derived from schizophrenic individuals, a potential concern is an inflated iMG enrichment for SCZ risk loci. However, we found no evidence of this, suggesting the observed $\mathrm{iMG}$ enrichment for $\mathrm{AD}$ risk loci was less likely to be confounded by using SCZ patient lines. Nonetheless, it would be ideal to use cell lines derived from healthy control subjects for such cell type-specific enrichment analyses for disease risk loci. Finally, although the iMG cells were transcriptionally indistinguishable from primary adult MGL cultured in vitro, that may not translate to functional equivalence with primary MGL under in vivo conditions. Co-culture of iMG with other types of neurons or astrocytes in $2 \mathrm{D}$ culture or as part of brain organoids may further enhance the validity of our in vitro model system.

The roles of MGL and the MGL-mediated neuroinflammation have been proposed in neurodegenerative disorders such as $\mathrm{AD}[6,7]$ and $\mathrm{PD}[33,34]$, and neuropsychiatric disorders such as SCZ [20], BIP [35], MDD $[36,37]$, and ASD [38]. Generating iMG that emulate adult MGL, either from hiPSC or from non-hiPSC cells [39], will have a broad impact by providing a renewable source of iMG for basic and clinical studies. Although iMG were not found enriched for major psychiatric genetic risk factors, these cells may remain to be a valuable cellular model for testing specific pathogenic hypotheses: MGL-mediated abnormal synaptic pruning in SCZ [20, 21 , and microglial activation in $\alpha$-synuclein aggregation in PD [33]. And more recent evidence [40, 41] supports microglial crosstalk with astrocytes - which did show disease enrichment across multiple disorders - a valuable future utility for advanced co-culture of induced cell types. In summary, our demonstration of the genetic relevance of hiPSC-derived iMG to AD offers support for iMG as an appropriate human cellular model for studying the enigmatic causal mechanisms of AD and understanding the role of neuroinflammatory factors in $\mathrm{AD}$ and other brain disorders, with the scalability to facilitate the development of more effective therapeutics.

\section{Methods}

hiPSC Lines

The two hiPSC lines, CD0000011 (CD11) and CD0000021 (CD21), were generated at RUCDR NIMH Stem Cell Center from the cryopreserved lymphoblasts of Molecular Genetics Study of Schizophrenia [42] by using the non-integrating Sendai virus
Butler III et al. 
method. The two lines are from SCZ cases, one male and the other female. The pluripotency of the hiPSC lines was confirmed by immunofluorescence staining of pluripotency markers and by Pluritest [43]. Confirmation of absence of chromosomal abnormality was performed by eSNP-karyotyping using RNA-seq data [44].

\section{Derivation of PMPs and $i M G$}

CD11 and CD21 hiPSC lines were used to generate iMG by Brownjohn's method [19]. These cell lines were cultured in mTeSR complete medium (85850, STEMCELL) with $2 \mathrm{~mL}$ daily media changes. At least 2 days after passaging, hiPSCs were passaged to single cells and plated at 10,000 cells per well in 96-well round bottom ultra-low attachment plates (Corning) in $100 \mu \mathrm{L} \mathrm{EB}$ media, and plates were centrifuged at $300 \mathrm{~g}$ for $3 \mathrm{~min}$. EB media was prepared by adding to the complete mTeSR media BMP-4 (Thermo Fisher), SCF (Thermo Fisher), VEGF-121 (Peprotech), and ROCK inhibitor (Tocris). Cells were cultured for 4 days, with half media change after 2 days. On day 4, 16 EBs were plated on a 6 -well plate and cultured in $3 \mathrm{~mL}$ hematopoietic media with $2 \mathrm{~mL}$ media exchanges every 5 days. Hematopoietic media was prepared by adding to the X-VIVO 15 (04-744Q, Lonza), GlutaMax (Thermo Fisher), Pen/strep (Thermo Fisher), $\beta$-mercaptoethanol (Sigma), M-CSF (Thermo Fisher), and IL-3 (Thermo Fisher). After 5 days culturing EBs in hematopoietic media, PMPs started to appear in the suspension and were produced continuously in suspension for 34 days. After 10 days of culturing EBs, PMPs could be harvested from suspension and plated in RPMI 1640 (Thermo Fisher) at 180,000 cells $/ \mathrm{cm}^{2}$ in 6 - or 12 -well plates. After $1 \mathrm{~h}, \mathrm{PMPs}$ adhered to surface and media was changed to complete MGL media. Full media changes were done every 2 days. Complete MGL media was prepared with RPMI 1640 by adding FBS (Thermo Fisher), Glutamax, Pen/strep, IL-24 (PeptroTech), and GM-CSF (PeproTech). Final differentiation of PMPs into MGL occurred over 6-10 days and cells could be kept for at least 1 month in this format.

\section{Phagocytosis of $i M G$}

To measure phagocytosis of bacterial particles, live imaging was performed on MGL exposed to $50 \mu \mathrm{g} / \mathrm{mL}$ pHrodo red conjugated E. coli bioparticles (Invitrogen). As a positive control for reduced phagocytosis, MGL were pre-treated (for $45 \mathrm{~min}$ ) and cotreated with $10 \mu \mathrm{M}$ of the actin polymerization inhibitor cytochalasin D. Live imaging was performed every 15 min after adding pHrodo E. coli in order to assess phagocytosis kinetics. Area of pHrodo signal in the fluorescent channel was measured and calculated as a proportion of total cell area measured in the DIC channel.

\section{Immunofluorescence Staining}

After washing cells in PBS, cell cultures were fixed in 4\% (w/ vol) paraformaldehyde and blocked with $1 \%$ BSA, 0.3\% (vol/vol) Triton-X100 in PBS before immunofluorescence staining. Primary antibodies were TREM2 N-terminal (R\&D Systems, 1:20), PU1 (Cell Signalling Technology, 1:100), and CD45 (Sigma, 1:200). Secondary antibodies were Alexa Fluor 488 chicken anti-goat (Thermo Fisher, 1:500) and Alexa Fluor 594 donkey anti-rabbit (Jackson ImmunoResearch, 1:1,000).

\section{Quantitative (Real-Time) PCR and Bulk RNA-seq}

Quantitative RT-PCR was performed on total RNA from each stem cell line after PBS wash. Cells were lysed in RLT plus buffer, and total RNA extracted with the RNeasy mini kit (Qiagen). cDNA was reverse transcribed from RNA with a high-capacity cDNA reverse transcription kit (Applied Biosystems). qPCR mix was prepared using Taqman Universal PCR master mix (Applied Biosystems), TaqMan mRNA assays (online suppl. Table 1), and performed on a Roche LightCycler 480. Total RNA was sent for sequencing to Novogene (en.novogene.com). Sequencing libraries were prepared using the NEB Nextera kit with customized adapters. We targeted the $25 \mathrm{M}, 2 \times 150$ bp pair-end reads per sample.

\section{Transcriptomic Analysis}

SRA archived raw sequencing reads were downloaded for NCBI Bioprojects (PRJNA350562, PRJNA435266, PRJEB22885, PRJNA496431) $[15,19,45,46]$ and processed alongside our sample reads. Reads were trimmed with Trimmomatic v0.36 [47] and mapped using STAR 2.6.1d [48]. Fragment counts were generated with featureCounts 1.6.3 [49]. Gene information was collected from biomaRt 2.38.0 [50], and 19,951 protein-coding genes with GC content information were used for downstream analysis. Overall principal component analysis utilized cqn 1.28.1 [51] for normalization and a minimum expression filter of at least $1 \mathrm{cpm}$ in at least 3 samples, applied with edgeR 3.24 .3 [52] resulting in a comparison set of 15,469 genes. The expression heatmap subset of ADrelevant genes, defined in Haenseler et al. [17], is derived from this normalized set. Pairwise sample distances were calculated from raw protein-coding gene counts using Poisson distance with PoiClaClu 1.0.2.1 [53].

\section{Cell Type Specificity}

Additional human neural cell data was gathered from Darmanis et al. [28] and merged with raw protein coding counts from above to generate a shared set of genes. Cell type specificity was generated for each sample set with EWCE 0.99.2 [54], after first removing sporadic expression with ANOVA analysis. Given the cell type similarity of all MGL and PMP sample sets (MGL-like sets), each of these seven sets was individually compared to all nonMGL-like sets, for a total of seven 9-sample specificity matrices. After examination of specificity distributions and MAGMA 1.07b [23] enrichment results, it was decided that enrichment values for non-MGL-like sets were comparable regardless of which MGLlike set they were tested against (online suppl. Fig. 3). For continuing analysis, the non-MGL-like specificity values when compared against primary adult MGL were chosen to generate gene sets for LDSC.

\section{Cell Type GWAS Enrichment Using MAGMA and LDSC}

MAGMA enrichment was conducted with the MAGMA Celltyping $\mathrm{R}$ package [27] (see URLs) using the specificity values above. Gene-level association statistics were tested with window sizes of $100 \mathrm{~kb}$ ( $50 \mathrm{~kb}$ upstream, $50 \mathrm{~kb}$ downstream), $57.5 \mathrm{~kb}$ (50 $\mathrm{kb}$ upstream, $7.5 \mathrm{~kb}$ downstream), and the recommended $11.5-\mathrm{kb}$ window ( $10 \mathrm{~kb}$ upstream, $1.5 \mathrm{~kb}$ downstream). The recommended window provided the best overall enrichment across all MGL-like cell types. GWAS datasets (online suppl. Table 4) for AD [6], PD [55], SCZ [56], MDD [57], BIP [58], ASD [59], and BMI [60] were run for each of the above 9-sample specificity matrices (online suppl. Fig. 3).
Genetic Relevance of iMG to AD and

Major Neuropsychiatric Disorders
Mol Neuropsychiatry 2019;5(suppl 1):85-96 DOI: $10.1159 / 000501935$ 
LDSC 1.0.0 [24, 25] analysis was conducted using gene sets derived from gene specificity values for each of the fifteen different cell types. Top percentile by specificity was checked at 10,20, 30, 40 , and $50 \%$ for all GWAS sets. Gene annotation files were constructed with the recommended $100-\mathrm{kb}$ window using the 1,000 Genomes Project phase 3 [61] data provided in the LDSC wiki guide (see URLs). The LDSC partitions were built with the recommended settings for a 1-cM window and HapMap3 limited SNPs. For regression, the baseline LD scores for 1,000 Genomes phase 3 version 2.2 were used with the provided HapMap3 SNP LD weights, and the "overlap-annot" argument was used with the 1,000 Genomes phase 3 minor-allele frequency files. The top $20 \%$ of genes provided the best overall enrichment across all MGL-like cell types and were used for figures (for all percentiles, see online suppl. Fig. 2).

\section{Accession Number}

Expression data for the iMG generated in this study have been submitted to the GEO database: GSE129630.

\section{URLS}

LDSC method: https://github.com/bulik/ldsc/wiki/Cell-typespecific-analyses; MAGMA: https://ctg.cncr.nl/software/magma; MAGMA Celltyping: https://github.com/NathanSkene/MAGMA_Celltyping; R: https://cran.r-project.org/; scripts used to process data, including software options available at: https://rbutleriii. github.io/center_for_psychiatric_genomics.

\section{Acknowledgements}

ThisworkwaspartiallysupportedbyNIHgrants(R01MH106575, R01MH116281, R01AG063175, and R21DA041600) and NorthShore pilot grant (to J.D.).

\section{Statement of Ethics}

The NorthShore University HealthSystem Institutional Review Board (IRB) approved the study.

\section{Disclosure Statement}

The authors have no competing interests to declare.

\section{Author Contributions}

A.K. performed the experiments, analyzed the data, and wrote the manuscript. R.R.B. analyzed data, performed bioinformatic analysis, and wrote the manuscript. S.Z. performed the initial analysis of the RNA-seq data. H.Z. and M.S. performed the lab experiments. A.R.S, K.L., Z.P.P., and P.V.G helped interpret the results and edited the manuscript. J.D. conceived the study, supervised the experiments and analyses, and wrote the manuscript.

\section{References}

1 Hardy J, Selkoe DJ. The amyloid hypothesis of Alzheimer's disease: progress and problems on the road to therapeutics. Science. $2002 \mathrm{Jul}$; 297(5580):353-6.

2 Hollingworth P, Harold D, Sims R, Gerrish A, Lambert JC, Carrasquillo MM, et al.; Alzheimer's Disease Neuroimaging Initiative; CHARGE consortium; EADI1 consortium. Common variants at ABCA7, MS4A6A/ MS4A4E, EPHA1, CD33 and CD2AP are associated with Alzheimer's disease. Nat Genet. 2011 May;43(5):429-35.

3 Naj AC, Jun G, Beecham GW, Wang LS, Vardarajan $\mathrm{BN}$, Buros J, et al. Common variants at MS4A4/MS4A6E, CD2AP, CD33 and EPHA1 are associated with late-onset Alzheimer's disease. Nat Genet. 2011 May;43(5):436-41.

4 Karch CM, Goate AM. Alzheimer's disease risk genes and mechanisms of disease pathogenesis. Biol Psychiatry. 2015 Jan;77(1):43-51.

5 Lambert JC, Ibrahim-Verbaas CA, Harold D, Naj AC, Sims R, Bellenguez C, et al.; European Alzheimer's Disease Initiative (EADI); Genetic and Environmental Risk in Alzheimer's Disease; Alzheimer's Disease Genetic Consortium; Cohorts for Heart and Aging Research in Genomic Epidemiology. Meta-analysis of 74,046 individuals identifies 11 new susceptibility loci for Alzheimer's disease. Nat Genet. 2013 Dec;45(12):1452-8.
6 Jansen IE, Savage JE, Watanabe K, Bryois J, Williams DM, Steinberg S, et al. Genome-wide meta-analysis identifies new loci and functional pathways influencing Alzheimer's disease risk. Nat Genet. 2019 Mar;51(3):404-13.

7 Sims R, van der Lee SJ, Naj AC, Bellenguez C, Badarinarayan N, Jakobsdottir J, et al.; ARUK Consortium; GERAD/PERADES, CHARGE, ADGC, EADI. Rare coding variants in PLCG2, $\mathrm{ABI} 3$, and TREM2 implicate microglial-mediated innate immunity in Alzheimer's disease. Nat Genet. 2017 Sep;49(9):1373-84.

8 Keren-Shaul H, Spinrad A, Weiner A, Matcovitch-Natan O, Dvir-Szternfeld R, Ulland TK, David E, Baruch K, Lara-Astaiso D, Toth B, Itzkovitz S, Colonna M, Schwartz M, Amit I: A Unique Microglia Type Associated with Restricting Development of Alzheimer's Disease. Cell. 2017 Jun 15;169(7):1276-1290.e17.

9 Galatro TF, Holtman IR, Lerario AM, Vainchtein ID, Brouwer N, Sola PR, et al. Transcriptomic analysis of purified human cortical microglia reveals age-associated changes. Nat Neurosci. 2017 Aug;20(8):1162-71.

10 Kierdorf K, Erny D, Goldmann T, Sander V, Schulz C, Perdiguero EG, et al. Microglia emerge from erythromyeloid precursors via Pu.1-and Irf8-dependent pathways. Nat Neurosci. 2013 Mar;16(3):273-80.
11 Ginhoux F, Greter M, Leboeuf M, Nandi S, See P, Gokhan S, et al. Fate mapping analysis reveals that adult microglia derive from primitive macrophages. Science. 2010 Nov; 330(6005):841-5

12 Prinz M, Priller J. Microglia and brain macrophages in the molecular age: from origin to neuropsychiatric disease. Nat Rev Neurosci. 2014 May;15(5):300-12.

13 Nayak D, Roth TL, McGavern DB. Microglia development and function. Annu Rev Immunol. 2014;32(1):367-402.

14 Muffat J, Li Y, Yuan B, Mitalipova M, Omer A, Corcoran S, et al. Efficient derivation of microglia-like cells from human pluripotent stem cells. Nat Med. 2016 Nov;22(11):1358-67.

15 Abud EM, Ramirez RN, Martinez ES, Healy LM, Nguyen CHH, Newman SA, Yeromin AV, Scarfone VM, Marsh SE, Fimbres C, Caraway CA, Fote GM, Madany AM, Agrawal A, Kayed R, Gylys KH, Cahalan MD, Cummings BJ, Antel JP, Mortazavi A, Carson MJ, Poon WW, Blurton-Jones M: iPSC-Derived Human Microglia-like Cells to Study Neurological Diseases. Neuron. 2017 Apr 19;94(2):278-293.e9.

16 Douvaras P, Sun B, Wang M, Kruglikov I, Lallos G, Zimmer M, et al. Directed Differentiation of Human Pluripotent Stem Cells to Microglia. Stem Cell Reports. 2017 Jun;8(6):1516-24. 
17 Haenseler W, Sansom SN, Buchrieser J, Newey SE, Moore CS, Nicholls FJ, et al. A Highly Efficient Human Pluripotent Stem Cell Microglia Model Displays a Neuronal-Co-culture-Specific Expression Profile and Inflammatory Response. Stem Cell Reports. 2017 Jun;8(6):1727-42.

18 Pandya H, Shen MJ, Ichikawa DM, Sedlock $A B$, Choi Y, Johnson KR, et al. Differentiation of human and murine induced pluripotent stem cells to microglia-like cells. Nat Neurosci. 2017 May;20(5):753-9.

19 Brownjohn PW, Smith J, Solanki R, Lohmann E, Houlden H, Hardy J, et al. Functional Studies of Missense TREM2 Mutations in Human Stem Cell-Derived Microglia. Stem Cell Reports. 2018 Apr;10(4):1294-307.

20 Sekar A, Bialas AR, de Rivera H, Davis A, Hammond TR, Kamitaki N, et al.; Schizophrenia Working Group of the Psychiatric Genomics Consortium. Schizophrenia risk from complex variation of complement component 4. Nature. 2016 Feb;530(7589):17783.

21 Sellgren CM, Gracias J, Watmuff B, Biag JD, Thanos JM, Whittredge PB, et al. Increased synapse elimination by microglia in schizophrenia patient-derived models of synaptic pruning. Nat Neurosci. 2019 Mar;22(3):37485.

22 Bachiller S, Jiménez-Ferrer I, Paulus A, Yang Y, Swanberg M, Deierborg T, et al. Microglia in Neurological Diseases: A Road Map to Brain-Disease Dependent-Inflammatory Response. Front Cell Neurosci. 2018 Dec;12: 488.

23 de Leeuw CA, Mooij JM, Heskes T, Posthuma D. MAGMA: generalized gene-set analysis of GWAS data. PLOS Comput Biol. 2015 Apr; 11(4):e1004219.

24 Bulik-Sullivan BK, Loh PR, Finucane HK, Ripke S, Yang J, Patterson N, et al.; Schizophrenia Working Group of the Psychiatric Genomics Consortium. LD Score regression distinguishes confounding from polygenicity in genome-wide association studies. Nat Genet. 2015 Mar;47(3):291-5.

25 Finucane HK, Bulik-Sullivan B, Gusev A, Trynka G, Reshef Y, Loh PR, et al.; ReproGen Consortium; Schizophrenia Working Group of the Psychiatric Genomics Consortium; RACI Consortium. Partitioning heritability by functional annotation using genome-wide association summary statistics. Nat Genet. 2015 Nov;47(11):1228-35.

26 Finucane HK, Reshef YA, Anttila V, Slowikowski K, Gusev A, Byrnes A, et al.; Brainstorm Consortium. Heritability enrichment of specifically expressed genes identifies disease-relevant tissues and cell types. Nat Genet. 2018 Apr;50(4):621-9.

27 Skene NG, Bryois J, Bakken TE, Breen G, Crowley JJ, Gaspar HA, et al.; Major Depressive Disorder Working Group of the Psychiatric Genomics Consortium. Genetic identification of brain cell types underlying schizophrenia. Nat Genet. 2018 Jun;50(6):825-33.
28 Darmanis S, Sloan SA, Zhang Y, Enge M, Caneda C, Shuer LM, et al. A survey of human brain transcriptome diversity at the single cell level. Proc Natl Acad Sci USA. 2015 Jun; 112(23):7285-90.

29 Tansey KE, Cameron D, Hill MJ. Genetic risk for Alzheimer's disease is concentrated in specific macrophage and microglial transcriptional networks. Genome Med. 2018 Feb;10(1):14.

30 Boyle EA, Li YI, Pritchard JK. An Expanded View of Complex Traits: From Polygenic to Omnigenic. Cell. 2017 Jun;169(7):1177-86.

31 Forrest MP, Zhang H, Moy W, McGowan H, Leites C, Dionisio LE, Xu Z, Shi J, Sanders AR, Greenleaf WJ, Cowan CA, Pang ZP, Gejman PV, Penzes P, Duan J: Open Chromatin Profiling in hiPSC-Derived Neurons Prioritizes Functional Noncoding Psychiatric Risk Variants and Highlights Neurodevelopmental Loci. Cell Stem Cell. 2017 Sep 7;21(3):305-318.e8.

32 Zhang S, Moy W, Zhang H, Leites C, McGowan $\mathrm{H}$, Shi J, et al. Open chromatin dynamics reveals stage-specific transcriptional networks in hiPSC-based neurodevelopmental model. Stem Cell Res (Amst). 2018 May;29:88-98.

33 Sampson TR, Debelius JW, Thron T, Janssen S, Shastri GG, Ilhan ZE, Challis C, Schretter CE, Rocha S, Gradinaru V, Chesselet MF, Keshavarzian A, Shannon KM, KrajmalnikBrown R, Wittung-Stafshede P, Knight R, Mazmanian SK: Gut Microbiota Regulate Motor Deficits and Neuroinflammation in a Model of Parkinson's Disease. Cell. 2016 Dec 1;167(6):1469-1480.e12.

34 Hirsch EC, Hunot S. Neuroinflammation in Parkinson's disease: a target for neuroprotection? Lancet Neurol. 2009 Apr;8(4):382-97.

35 Haarman BC, Riemersma-Van der Lek RF, de Groot JC, Ruhé HG, Klein HC, Zandstra TE, et al. Neuroinflammation in bipolar disorder - A [(11)C]-(R)-PK11195 positron emission tomography study. Brain Behav Immun. 2014 Aug;40:219-25

36 Hurley LL, Tizabi Y. Neuroinflammation, neurodegeneration, and depression. Neurotox Res. 2013 Feb;23(2):131-44.

37 Tang MM, Lin WJ, Pan YQ, Li YC. Fibroblast Growth Factor 2 Modulates Hippocampal Microglia Activation in a Neuroinflammation Induced Model of Depression. Front Cell Neurosci. 2018 Aug;12:255.

38 Suzuki K, Sugihara G, Ouchi Y, Nakamura K, Futatsubashi M, Takebayashi K, et al. Microglial activation in young adults with autism spectrum disorder. JAMA Psychiatry. 2013 Jan;70(1):49-58.

39 Sellgren CM, Sheridan SD, Gracias J, Xuan D, Fu T, Perlis RH. Patient-specific models of microglia-mediated engulfment of synapses and neural progenitors. Mol Psychiatry. 2017 Feb;22(2):170-7.

40 Rothhammer V, Borucki DM, Tjon EC, Takenaka MC, Chao CC, Ardura-Fabregat A, et al. Microglial control of astrocytes in response to microbial metabolites. Nature. 2018 May; 557(7707):724-8.
41 Liddelow SA, Guttenplan KA, Clarke LE, Bennett FC, Bohlen CJ, Schirmer L, et al. Neurotoxic reactive astrocytes are induced by activated microglia. Nature. 2017 Jan;541(7638): 481-7.

42 Shi J, Levinson DF, Duan J, Sanders AR, Zheng $\mathrm{Y}$, Pe'er I, et al. Common variants on chromosome $6 \mathrm{p} 22.1$ are associated with schizophrenia. Nature. 2009 Aug;460(7256):753-7.

43 Müller FJ, Schuldt BM, Williams R, Mason D, Altun G, Papapetrou EP, et al. A bioinformatic assay for pluripotency in human cells. Nat Methods. 2011 Apr;8(4):315-7.

44 Weissbein U, Schachter M, Egli D, Benvenisty $\mathrm{N}$. Analysis of chromosomal aberrations and recombination by allelic bias in RNA-Seq. Nat Commun. 2016 Jul;7(1):12144.

45 Buskin A, Zhu L, Chichagova V, Basu B, Mozaffari-Jovin S, Dolan D, et al. Disrupted alternative splicing for genes implicated in splicing and ciliogenesis causes PRPF31 retinitis pigmentosa. Nat Commun. 2018 Oct; 9(1):4234.

46 Hadjicharalambous MR, Roux BT, FeghaliBostwick CA, Murray LA, Clarke DL, Lindsay MA. Long Non-coding RNAs Are Central Regulators of the IL-1 $\beta$-Induced Inflammatory Response in Normal and Idiopathic Pulmonary Lung Fibroblasts. Front Immunol. 2018 Dec;9:2906.

47 Bolger AM, Lohse M, Usadel B. Trimmomatic: a flexible trimmer for Illumina sequence data. Bioinformatics. 2014 Aug;30(15):2114-20.

48 Dobin A, Davis CA, Schlesinger F, Drenkow J, Zaleski C, Jha S, et al. STAR: ultrafast universal RNA-seq aligner. Bioinformatics. 2013 Jan;29(1):15-21.

49 Liao Y, Smyth GK, Shi W. featureCounts: an efficient general purpose program for assigning sequence reads to genomic features. Bioinformatics. 2014 Apr;30(7):923-30.

50 Durinck S, Spellman PT, Birney E, Huber W. Mapping identifiers for the integration of genomic datasets with the $\mathrm{R} /$ Bioconductor package biomaRt. Nat Protoc. 2009;4(8): 1184-91.

51 Hansen KD, Irizarry RA, Wu Z. Removing technical variability in RNA-seq data using conditional quantile normalization. Biostatistics. 2012 Apr;13(2):204-16.

52 Robinson MD, McCarthy DJ, Smyth GK edgeR: a Bioconductor package for differential expression analysis of digital gene expression data. Bioinformatics. 2010 Jan;26(1):139-40.

53 Witten DM. Classification and clustering of sequencing data using a Poisson model. Ann Appl Stat. 2011;5(4):2493-518.

54 Skene NG, Grant SG. Identification of Vulnerable Cell Types in Major Brain Disorders Using Single Cell Transcriptomes and Expression Weighted Cell Type Enrichment. Front Neurosci. 2016 Jan; 10:16.

55 Nalls MA, et al.; International Parkinson's Disease Genomics Consortium. Expanding Parkinson's disease genetics: novel risk loci, genomic context, causal insights and heritable risk. bioRxiv. 2019.
Genetic Relevance of iMG to AD and Major Neuropsychiatric Disorders
Mol Neuropsychiatry 2019;5(suppl 1):85-96 DOI: $10.1159 / 000501935$ 
56 Schizophrenia Working Group of the Psychiatric Genomics Consortium. Biological insights from 108 schizophrenia-associated genetic loci. Nature. 2014 Jul;511(7510):421-7.

57 Wray NR, Ripke S, Mattheisen M, Trzaskowski M, Byrne EM, Abdellaoui A, et al.; eQTLGen; 23andMe; Major Depressive Disorder Working Group of the Psychiatric Genomics Consortium. Genome-wide association analyses identify 44 risk variants and refine the genetic architecture of major depression. Nat Genet. 2018 May;50(5):668-81.
58 Stahl EA, Breen G, Forstner AJ, McQuillin A, Ripke S, Trubetskoy V, et al. Genome-wide association study identifies 30 Loci Associated with Bipolar Disorder. bioRxiv. 2018: 173062.

59 Grove J, Ripke S, Als TD, Mattheisen M, Walters RK, Won H, et al.; Autism Spectrum Disorder Working Group of the Psychiatric Genomics Consortium; BUPGEN; Major Depressive Disorder Working Group of the Psychiatric Genomics Consortium; 23andMe Research Team. Identification of common genetic risk variants for autism spectrum disorder. Nat Genet. 2019 Mar;51(3):431-44.
60 Yengo L, Sidorenko J, Kemper KE, Zheng Z, Wood AR, Weedon MN, et al.; GIANT Consortium. Meta-analysis of genome-wide association studies for height and body mass index in 700000 individuals of European ancestry. Hum Mol Genet. 2018 Oct;27(20): 3641-9.

611000 Genomes Project Consortium, Auton A, Brooks LD, Durbin RM, Garrison EP, Kang HM, Korbel JO, Marchini JL, McCarthy S, McVean GA, Abecasis GR. A global reference for human genetic variation. Nature. 2015; 526(7571):68-74. 